\title{
Hybrid Management of an Ulnar Artery Pseudoaneurysm Postphlebotomy
}

\author{
Heather K. Moriarty, ${ }^{1,0}$ Peter Charalabidis ${ }^{2} \quad$ Korana Musicki ${ }^{2} \quad$ Tuan Phan $^{1} \quad$ Tim Joseph ${ }^{1}$ Warren Clements ${ }^{1}$
}

\author{
${ }^{1}$ Department of Radiology, Alfred Hospital, Melbourne, Victoria, \\ Australia \\ 2Department of Vascular Surgery, Alfred Hospital, Melbourne, \\ Victoria, Australia
}

\begin{abstract}
Address for correspondence Heather Kate Moriarty, MB BCh, BAO, MMedSci, Department of Radiology, Alfred Hospital, 55 Commercial Rd, Melbourne, Victoria 3004, Australia (e-mail: heather.moriarty@gmail.com).
\end{abstract}
Abstract
Keywords
- pseudoaneurysm
- ulnar artery
- endovascular
- stent graft
- phlebotomy

The purpose of this case report is to describe the occurrence and management of an ulnar artery pseudoaneurysm post phlebotomy. A 36-year-old worker with progressively painful nondominant forearm swelling was referred at 4 weeks postphlebotomy. Serial imaging showed an expanding proximal ulnar pseudoaneurysm. This was managed with a covered stent in the ulnar artery and coiling of the interosseous artery. The hematoma was evacuated, and the neurovascular bundle was protected with muscle coverage. At follow-up, the stent was patent, and there was no distal ischemia. Pseudoaneurysms post phlebotomy are rare. We demonstrate successful treatment of an ulnar artery pseudoaneurysm with stent graft and coiling of the interosseous branch.

\section{Introduction}

Pseudoaneurysm formation is a recognized complication of iatrogenic and noniatrogenic penetrating and blunt trauma, as well as infection..$^{1,2}$ Endovascular correction is appealing due to its minimally invasive nature, the potential to treat quickly, and allay immediate danger of rupture or thromboembolization, and the resultant life- or limb-threatening hemorrhage or ischemia.

Brachial artery pseudoaneurysms have been described in the setting of dialysis access and postphlebotomy, but they rarely occur in the ulnar artery. ${ }^{3-5}$ Ulnar artery pseudoaneurysms are most frequently managed by surgery, which may involve repair or ligation. ${ }^{6,7}$ Coil embolization of an ulnar artery pseudoaneurysm has been described, and a single case report exists of exclusion of an ulnar artery pseudoaneurysm using a stent graft. ${ }^{8,9}$

\section{Case Report}

A 36-year-old right-handed office worker underwent routine blood testing taken from the proximal volar aspect of the left upper forearm. Following a "forceful sneeze" several days later, he experienced sudden forearm swelling and pain. Despite repeated primary medical attendance, specialist

received

April 24, 2019

accepted after revision

May 20, 2019

published online

December 4, 2019 referral was delayed until diagnosis of a pseudoaneurysm by duplex ultrasound on day 11 and confirmation by computed tomography (CT) angiography on day 21 . On presentation at nearly 4-weeks postphlebotomy, the left forearm had pain and swelling, with heavy bruising but no erythema or warmth. Despite a mild fixed flexion deformity and paresthesia in the middle three fingers, there was no compartment tightness or objective peripheral neurologic deficit. Upper limb pulses were all preserved, with good perfusion of his hand and fingers, and Allen's test was negative (there was adequate circulation to the hand on manual occlusion of the radial or ulnar artery). His hematological and biochemical work-up was negative for evidence of infection.

Computed tomography showed a complex $35-\mathrm{mm}$ proximal ulnar artery pseudoaneurysm ( - Fig. 1 ), which had increased in size from $18 \mathrm{~mm}$, as documented on the ultrasound 10 days prior. At multidisciplinary discussion, an endovascular approach was favored due to concerns that surgical exploration would carry a reasonable risk of median nerve injury some 4 weeks after the initial insult. Treatment was considered urgent given the fixed flexion deformity and demonstrated rapid expansion with a possible risk of rupture in a $35-\mathrm{mm}$ pseudoaneurysm.

Access was obtained through the right femoral artery. A 5-Fr 90-cm Guidecath (Codman Neuro) was placed in
License terms

Vascular and Interventional Radiology

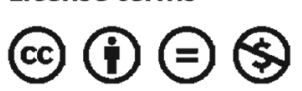




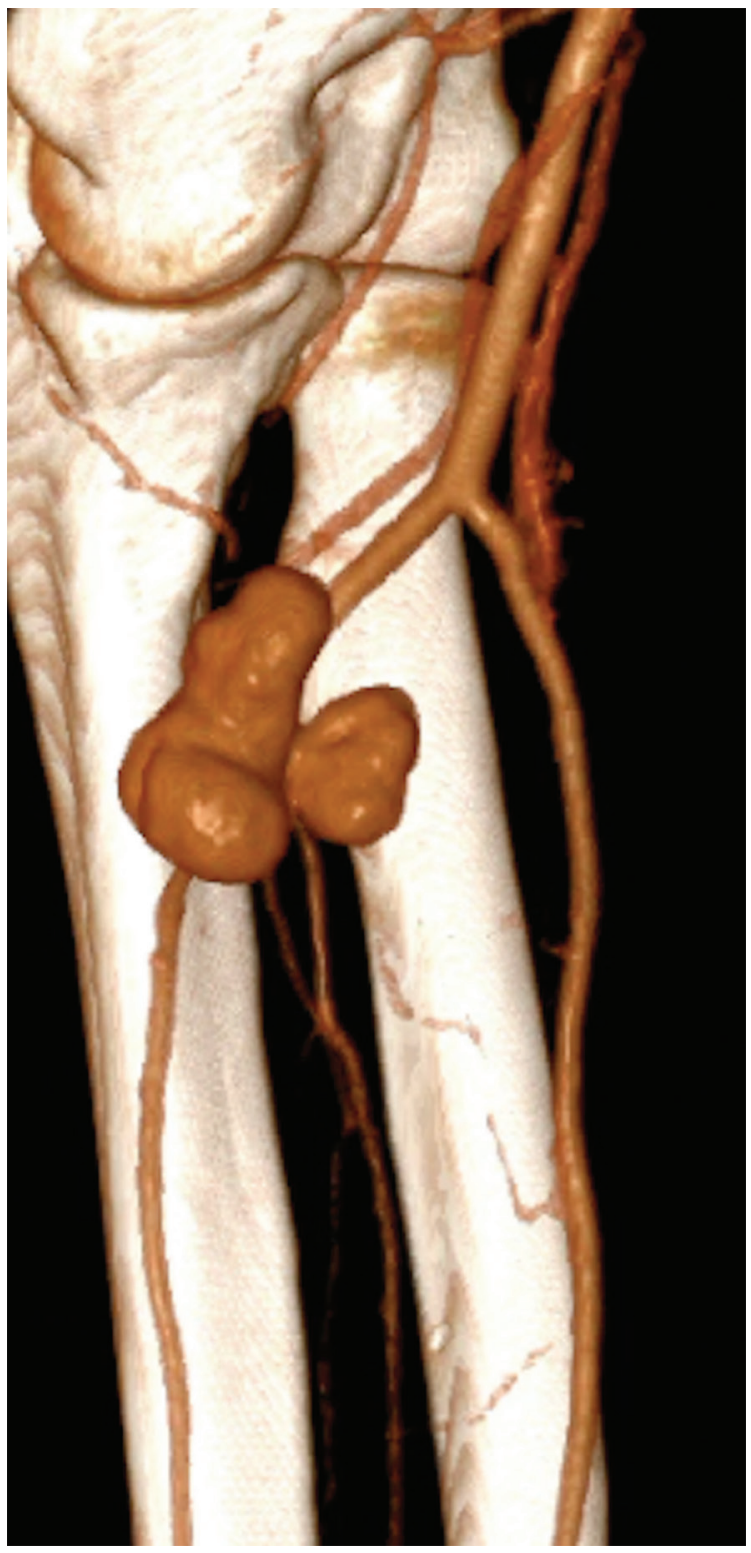

Fig. 1 Volume-rendered computed tomography (CT) imaging demonstrates a complex $35-\mathrm{mm}$ pseudoaneurysm arising from the proximal ulnar artery with involvement of the interosseous artery.

the distal brachial artery. Angiography demonstrated the pseudoaneurysm arising from the ulnar artery, with poor antegrade filling of the ulnar and common interosseous arteries distally ( - Fig. 2 a). The main inflow to the hand was through the radial artery, and with the palmar arch intact, there was retrograde flow in the distal ulnar artery. On-table ultrasound demonstrated a 14-mm longitudinal transection producing a long and broad neck ( - Fig. 2b). A Progreat microcatheter (Terumo) was used to selectively catheterize and coil embolize the anterior, posterior, and common interosseous arteries. The ulnar artery distal to the lesion was then selected, and two BeGraft stents, $2.5 \times 21 \mathrm{~mm}$ and $2.5 \times 12 \mathrm{~mm}$ (Bentley Innomed $\mathrm{GmbH}$ ), were deployed and postdilated to the rated burst pressure ( - Fig. 3a). Two covered stents were used to extend the covered length and decrease the risk of type 1 endoleak, as a single longer stent was not available to the interventional radiologists. Completion angiography demonstrated antegrade flow in the ulnar artery and no further filling of the pseudoaneurysm sac ( - Fig. $\mathbf{3 b}$ ).

On day 1 postendovascular exclusion, the patient underwent surgical exploration. The hematoma was evacuated with the stented ulnar artery on view at the base, and no arterial bleeding was seen. No evidence of infection such as a purulent collection was seen, and subsequent culture of the hematoma was negative. Adjacent muscle tissue was mobilized and secured to cover the neurovascular bundle.

The patient was discharged 2 days later on a regimen of $100 \mathrm{mg}$ of aspirin and $75 \mathrm{mg}$ of clopidogrel daily. Follow-up at 2 weeks postprocedure demonstrated an improved range of movement and palpable radial and ulnar pulses. Duplex ultrasound demonstrated persisting antegrade flow in the ulnar artery.

\section{Discussion}

Accidental arterial puncture is a recognized complication of venous access. The most common outcome is localized hematoma. Rarer sequelae include arteriovenous fistula, pseudoaneurysm formation, and compartment syndrome. ${ }^{10}$ Imaging with ultrasound, CT, and/or catheter angiography is necessary to confirm the diagnosis and delineate the anatomy, including the complexity and size of the neck, which impacts management options.

Options for the management of pseudoaneurysms vary depending on the location, size, anatomy, and acuity. Embolization, either endovascular or percutaneous, is often favored as it is minimally invasive and allows access to the lesion without disrupting the neurovascular bundle. Stent grafts may also be used, particularly for pseudoaneurysms originating from the major arteries or for those with larger necks, with the advantage of allowing antegrade perfusion of the distal organ. A combination of both techniques can be used, such as in this case, to preserve flow with covered stent placement in the most important vessel and to exclude smaller contributing vessels, thus preventing endoleak. This initial venepuncture attempt was in the upper forearm remote from the flexion point and thus allowed safe stent graft placement without concern for stent fracture. If the stent were to occlude, the flow dynamics of the forearm will be similar to the initial presentation-radial artery flow to the hand, intact palmar arch, and likely retrograde flow in the distal ulnar.

In this case, the hybrid approach was undertaken based on several features of the presentation that made surgery alone less favorable. The anatomy with a $14-\mathrm{mm}$ longitudinal rent in a $2.5-\mathrm{mm}$-diameter vessel meant that a simple repair was improbable; ligation would be likely, and although patching or bypassing could be considered, in such small vessel it would likely occlude early despite the effort to preserve flow. The timing at 4 weeks was anticipated to be met with significant inflammation of adjacent structures tantamount to a redo exposure, which, combined with the need to mobilize a longer section of the ulnar artery, its location at depth, 


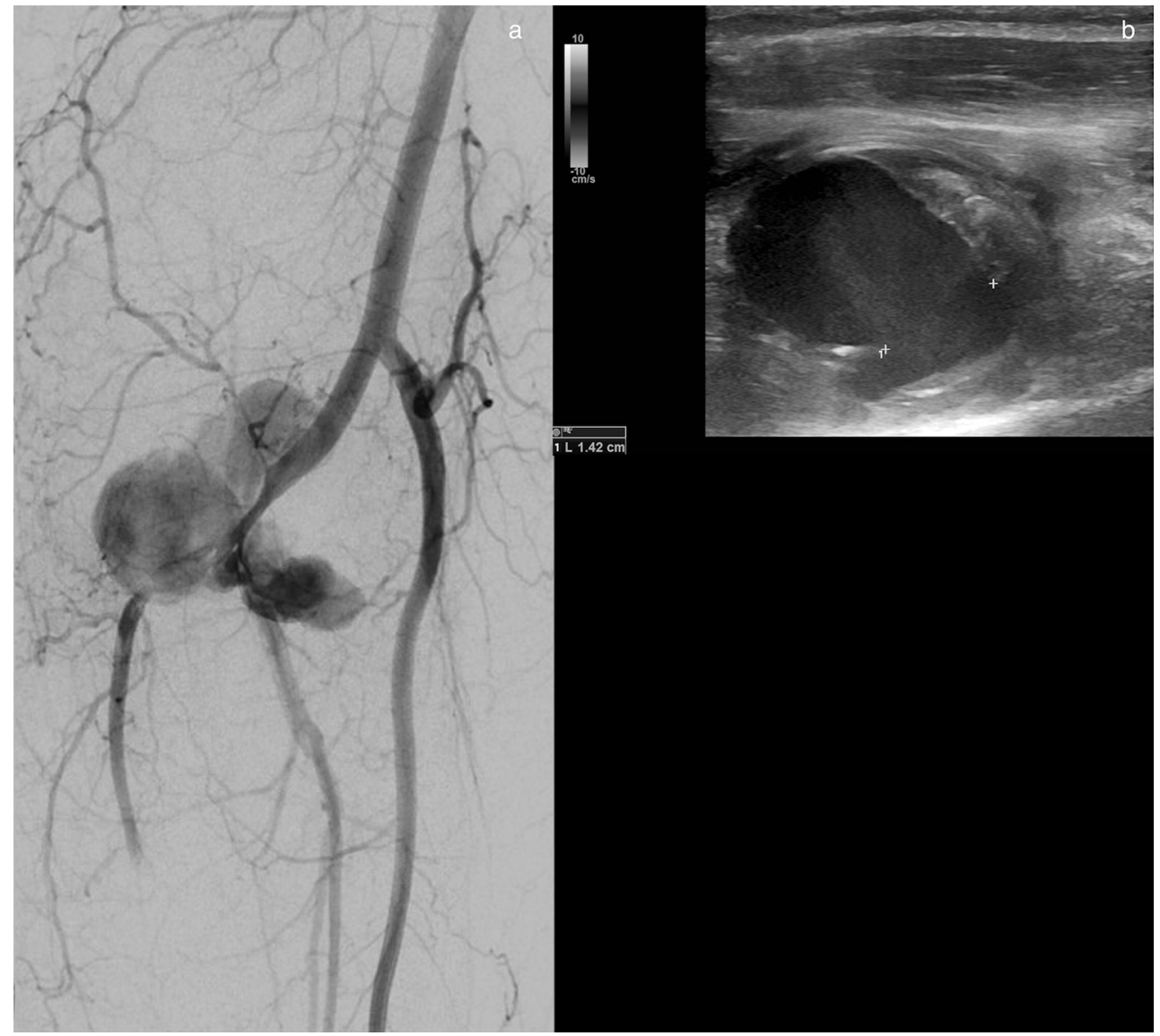

Fig. 2 (a) Angiography demonstrates a 35-mm multilobed pseudoaneurysm arising from the ulnar artery, with poor antegrade filling of the ulnar and interosseous arteries. (b) On-table ultrasound demonstrated a 14-mm neck arising from the ulnar artery.

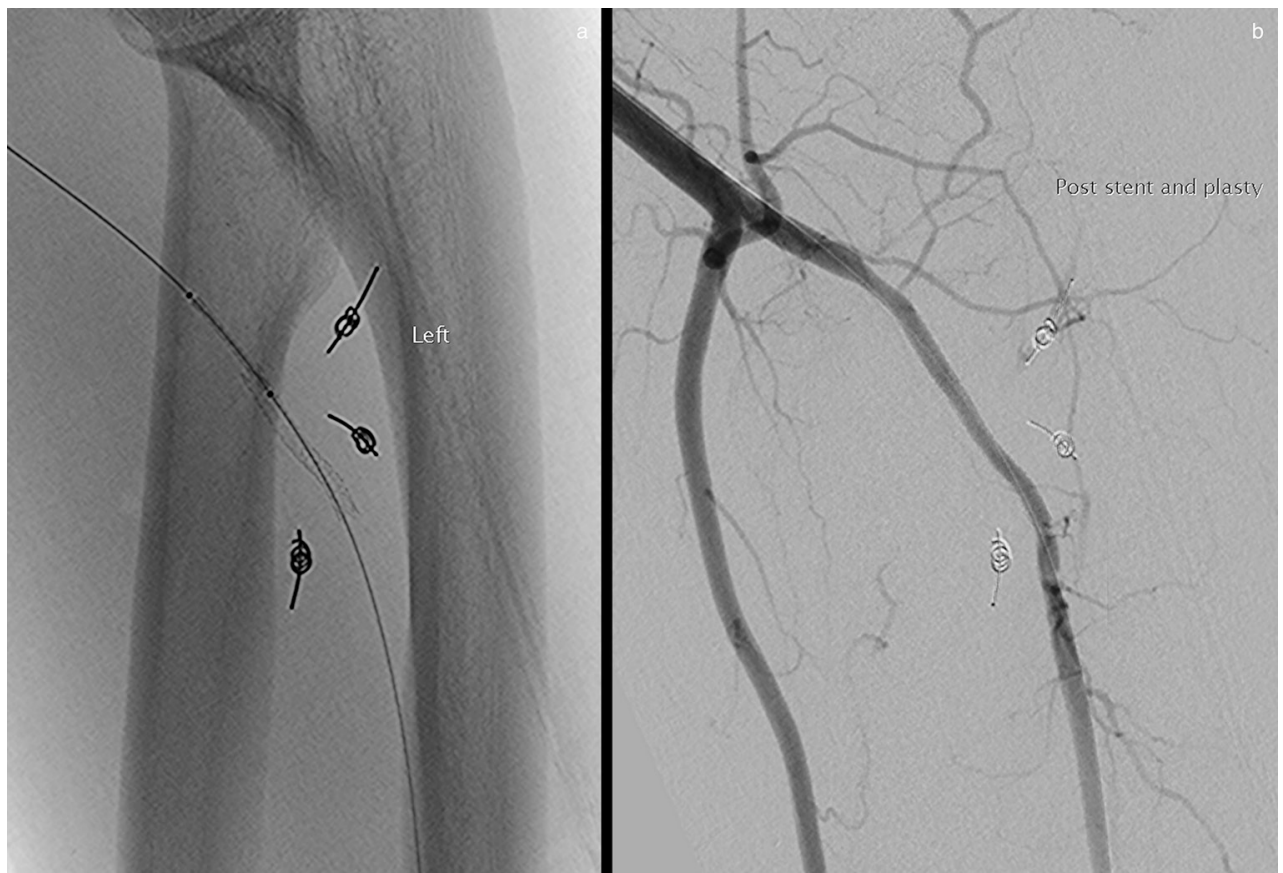

Fig. 3 (a) The interosseous artery branches are coil embolized, and overlapping stent grafts are deployed in the ulnar artery covering the aneurysm neck. (b) Completion angiography demonstrated antegrade flow in the ulnar artery and no pseudoaneurysm filling. 
and its proximity to the median nerve, led to a higher risk of nerve injury.

Instead, our hybrid approach provided rapid exclusion of the pseudoaneurysm with its evolving sequelae. It allowed for safer surgical exploration including ensuring that the surgical field was free of active bleeding and obviated the need for vascular mobilization in scarred tissue. It also allowed the reestablishment of antegrade flow to the hand through the injured ulnar artery in a young patient rather than sacrificing the artery, as might be the traditional approach. Endovascular management necessarily increases the associated cost, most notably due to the use of the endovascular equipment. This approach is feasible in institutions where timely access to an interventional radiology service with appropriate equipment is available.

\section{Conclusion}

This is an unusual occurrence of an ulnar artery pseudoaneurysm postphlebotomy. A system where good quality imaging can be obtained and decisions are made at a multidisciplinary level allows for all options to be explored and a consensus to be achieved. In this case, the stent graft allowed rapid exclusion of the pseudoaneurysm and re-establishment of antegrade flow to the hand through the ulnar artery, allowing safer and quicker surgical exploration and hematoma evacuation, as well as a favorable outcome for the patient. Although long-term patency is yet to be established, treatment in the acute setting using our approach was safe and rapidly effective.

\section{Funding}

This study was not funded.

\section{Ethical Approval}

All procedures performed in studies involving human participants were in accordance with the ethical standards of the institutional and/or national research committee and with the 1964 Helsinki declaration and its later amendments or comparable ethical standards. The study was performed in accordance with guidelines from the Alfred Hospital Research and Ethics Committee. This material is not under submission elsewhere.

\section{Consent}

Informed consent was obtained from all individual participants included in the study. Consent for publication was obtained for every individual person's data included in the study.

\section{Conflict of Interest}

The authors declare that there is no financial arrangement or other relationship that could be construed as a conflict of interest.

\section{References}

1 Saad NE, Saad WE, Davies MG, Waldman DL, Fultz PJ, Rubens DJ. Pseudoaneurysms and the role of minimally invasive techniques in their management. Radiographics 2005;25(Suppl 1):S173-S189

2 Stolt M, Braun-Dullaeus R, Herold J. Do not underestimate the femoral pseudoaneurysm. Vasa 2018;47(3):177-185

3 Yildirim S, Nursal TZ, Yildirim T, Tarim A, Caliskan K. Brachial artery pseudoaneurysm: a rare complication after haemodialysis therapy. Acta Chir Belg 2005;105(2):190-193

4 Popovsky MA, McCarthy S, Hawkins RE. Pseudoaneurysm of the brachial artery: a rare complication of blood donation. Transfusion 1994;34(3):253-254

5 Santini E, Ferri F, Roncolini G, Serafini L. Iatrogenic false aneurysm of the ulnar artery. A clinical case and review of the literature [in Italian]. Minerva Cardioangiol 1997;45(12):615-619

6 Hurst SA, Raveendran S, Eckersley JR. Ulnar artery pseudoaneurysm following single non-penetrating trauma to the hypothenar region. J Hand Surg Eur Vol 2014;39(7):786-788

7 Sekino S, Takagi H, Kato T, Matsuno Y, Sekido Y, Umemoto T. Nontraumatic pseudoaneurysm of the proximal ulnar artery with eosinophilia. J Vasc Surg 2005;42(6):1233-1235

8 Woodley-Cook J, Konieczny M, Simons M. The ulnar artery pseudoaneurysm. BMJ Case Rep 2015;2015:bcr201521791

9 Nough H, Bagherinasab M, Emami M, Sarebanhassanabadi M, Hadiani L. Endovascular treatment of post-traumatic pseudoaneurysms of ulnar and radial artery. Acta Med Iran 2014;52(11):865-867

10 Morgan R, Belli AM. Current treatment methods for postcatheterization pseudoaneurysms. J Vasc Interv Radiol 2003; 14(6):697-710 